\title{
Estudo preliminar dos fatores associados a quedas em idosos
}

\author{
Preliminary study of the associated factors with falls in the elderly \\ Estudio preliminar de los factores asociados con caídas en ancianos
}

\section{Resumo}

Introdução: A queda é um problema de saúde pública e a identificação dos fatores associados constitui importante estratégia na prevenção da saúde. Objetivo: Identificar a frequência de quedas e fatores associados entre idosos adscritos a uma Unidade Básica de Saúde (UBS). Metodologia: Estudo transversal realizado em UBS do município de Petrolina, PE (Brasil) envolvendo 40 idosos com idade $\geq 60$ anos. Para avaliação socioeconômica, estado geral de saúde, histórico de quedas e medo de cair foram utilizados questionários estruturados. O Mini Exame do Estado Mental (MEEM) foi utilizado como indicativo da função cognitiva e para avaliação do risco de queda utilizou-se o Timed Up And Go (TUG). Em adição, realizou-se avaliação antropométrica. Foram calculadas a distribuição das frequências absolutas e relativas, e os intervalos de confiança de 95\% (IC95\%). Associações entre a ocorrência de queda e as demais variáveis foram calculadas por meio dos testes Qui-Quadrado ou Exato de Fisher. Resultados: Foram incluídos 38 idosos, com mediana de idade 68,5 anos, 42,1\% (n=16) apresentaram histórico de pelo menos uma queda nos últimos 12 meses e 21,1\% $(n=8)$ deixaram de realizar alguma atividade por medo de cair. Não foram verificadas associações significativas entre ocorrência de queda e as variáveis idade, sexo, diabetes mellitus, hipertensão arterial sistêmica, risco de queda, medo de cair, déficit visual e doença osteoarticular. Conclusão: A frequência de quedas foi elevada entre os idosos analisados e não foi observada associação significativa entre ocorrência de queda e os fatores preditores analisados.

Palavras-chave: Idoso; Saúde do Idoso; Fatores de Risco; Morbidade. 


\begin{abstract}
Introduction: Fall is a public health problem and the identification of associated factors constitutes an important strategy in health prevention. Objective: To identify the frequency of falls and associated factors among elderly people enrolled in a Basic Health Unit (BHU). Methodology: A cross-sectional study carried out in BHU in the city of Petrolina, PE (Brazil) involving 40 elderly people aged $\geq 60$ years. Socioeconomic assessment, general health status, and history of falls, and fear of falling were assessed by structured questionnaires. The Mini-Mental State Examination (MMSE) was used as an indicator of cognitive function and the Timed Up and Go (TUG) was used to assess the risk of fall. An anthropometric assessment was also performed. The distribution of absolute and relative frequencies was calculated with confidence intervals at 95\% (CI95\%). Associations between the occurrence of falls and the other variables were calculated using the Chi-square or Fisher's exact tests. Results: 38 elderly people were included, with a median age of 68.5 years, $42.1 \%(n=16)$ had a history of at least one fall in the last 12 months and $21.1 \%(n=8)$ failed to perform any activity for fear of falling. There were no significant associations between the occurrence of falls and the variables age, sex, diabetes mellitus, systemic arterial hypertension, risk of fall, fear of falling, visual impairment, and osteoarticular disease. Conclusion: The frequency of falls was high among the elderly analyzed and no significant associations were verified between the occurrence of falls and the predictive factors.
\end{abstract}

Keywords: Aged; Health of the Elderly; Risk Factors; Morbidity.

\title{
Resumen
}

Introducción: La caída es un problema de salud pública y la identificación de factores asociados constituye una estrategia importante en la prevención sanitaria. Objetivo: Identificar la frecuencia de caídas y los factores asociados entre los ancianos inscritos en una Unidad Básica de Salud (UBS). Metodología: Estudio transversal realizado en UBS en la ciudad de Petrolina, PE (Brasil) que involucró a 40 ancianos $\geq 60$ años. La evaluación socioeconómica, el estado general de salud, los antecedentes de caídas y miedo a caer se evaluaron mediante cuestionarios estructurados. El Mini Examen del Estado Mental (MEEM) se utilizó como indicador de la función cognitiva y el Timed Up and Go (TUG) se utilizó para evaluar el riesgo de caída. También se realizó una evaluación antropométrica. La distribución de frecuencias absolutas y relativas se calculó con intervalos de confianza al 95\% (IC95\%). Las asociaciones entre la ocurrencia de caídas y el resto de las variables se calcularon mediante la prueba de Chi-cuadrado o la prueba exacta de Fisher. Resultados: Se incluyeron 38 ancianos, con una mediana de edad de 68,5 años, el 42,1\% (n=16) tenía antecedente de al menos una caída en los últimos 12 meses y el 21,1\% (n=8) dejó de realizar alguna actividad por miedo a caer. No se encontraron asociaciones significativas entre la ocurrencia de caídas y las variables edad, sexo, diabetes mellitus, hipertensión arterial sistémica, riesgo de caída, miedo a caer, discapacidad visual y enfermedad osteoarticular. Conclusión: La frecuencia de caídas fue alta entre los ancianos analizados y no se verificaron asociaciones significativas entre la ocurrencia de caídas y los factores predictivos.

Palabras clave: Anciano; Salud del Anciano; Factores de Riesgo; Morbilidad.

\section{Introdução}

A população mundial tem apresentado alterações na sua estrutura etária, uma vez que o número de idosos tem crescido em quase todas as regiões do mundo (Santos et al., 2013). A população brasileira destaca-se, nesse contexto, por apresentar um processo de envelhecimento mais rápido quando comparada a população mundial (Miranda et al., 2016; Santos et al., 2015). Essa mudança na estrutura etária reforça a necessidade de políticas públicas voltadas à melhor qualidade de vida dessa população (Miranda et al., 2016).

Sabe-se que o processo de envelhecimento é acompanhado de alterações fisiológicas e funcionais importantes como a redução da acuidade visual, diminuição de força muscular e do equilíbrio, aumentando o risco de quedas e as lesões decorrentes destas entre os idosos (Gamage et al., 2019, Rodrigues et al., 2014; Texeira et al., 2019).

A queda é considerada um evento multifatorial que envolve fatores intrínsecos ou extrínsecos que afetam a estabilidade, sendo definida como um deslocamento não intencional do corpo para um nível inferior à posição inicial (Nascimento \& Tavares, 2016; Santos et al., 2015, Texeira et al., 2019; Vieira et al., 2018). Esse evento é um problema de saúde pública em muitos países (Bekibele et al., 2010; Gamage et al., 2019), uma vez que a maioria das quedas pode ocasionar consequências preocupantes para idosos, tais como, incapacidade e lesões graves (Prato et al., 2017).

No Brasil a ocorrência de quedas entre idosos é elevada. Dados obtidos por meio de um estudo populacional, no qual foram avaliados idosos de 23 estados brasileiros, demonstraram uma prevalência de quedas de 27,6\% (Siqueira et al., 2011). Outros estudos que avaliaram idosos em diferentes cidades do país evidenciaram taxas de prevalência entre $28,1 \%$ e $53,6 \%$ 
(Motta et al., 2010; Nascimento \& Tavares, 2016; Santos et al., 2015; Viera et al., 2018). Outro dado importante é o medo de cair, que afeta até $85 \%$ dos idosos (Scheffer et al., 2008), interferindo nas atividades de vida diária, assim como no bem-estar físico e psicossocial dos indivíduos (Rivasi et al., 2020).

Diversos fatores podem estar associados às quedas na população idosa, como, idade, sexo, comorbidades, uso de medicamentos, distúrbios do sono e disfunções do equilíbrio e da marcha (Gamage et al., 2019). A identificação dos fatores associados às quedas, sobretudo os modificáveis, é de fundamental importância pois pode auxiliar na elaboração de estratégias preventivas e de promoção da saúde para os idosos, favorecendo autonomia e independência funcional (Motta et al., 2010), uma vez que ainda são necessários mecanismos que consolidem o modelo de atenção à saúde do idoso (Miranda et al., 2016).

Diante do exposto, o objetivo do presente estudo foi identificar a frequência de quedas e os fatores associados entre idosos adscritos a uma Unidade Básica de Saúde (UBS) do município de Petrolina, PE, Brasil.

\section{Metodologia}

Trata-se de um estudo preliminar (Pereira et al, 2018), de caráter descritivo e analítico, com delineamento transversal realizado em uma Unidade Básica de Saúde (UBS) da zona urbana do município de Petrolina, PE, Brasil. O estudo foi conduzido entre outubro e novembro de 2018, período em que a referida UBS contava com 1.601 idosos adscritos. A amostra não probabilística e voluntária envolveu 40 indivíduos com 60 anos ou mais de idade (idosos).

Esta pesquisa foi aprovada pelo Comitê de Ética em Pesquisa da Faculdade de Ciências Médicas de Pernambuco (CEP-FCM/PE) sob parecer número 2.753.501 e Certificado de Apresentação de Apreciação Ética (CAAE) número 88147918.9.000.5192. Este estudo obedeceu a todos os preceitos das Resoluções 466/2012 e 510/2016 do Conselho Nacional de Saúde e todos os participantes assinaram termo de consentimento livre e esclarecido (TCLE).

Para seleção dos participantes foram adotados os seguintes critérios de inclusão: ter idade igual ou superior a 60 anos; ser funcionalmente ativo e apresentar função cognitiva preservada. Definiu-se como atividade funcional adequada a capacidade de caminhar dentro e fora de casa sem dificuldade ou ajuda externa e função cognitiva preservada foi definida como auto capacidade para interagir com o entrevistador (Almeida-Santos et al., 2016).

Os critérios de exclusão foram: pontuação menor que os pontos de corte por escolaridade no Mini Exame do Estado Mental (MEEM), que indica má função cognitiva (Brucki et al., 2003) e a não conclusão do preenchimento dos questionários.

Para avaliação socioeconômica foi utilizado um questionário estruturado, com questões acerca dos dados sociodemográficos (idade, estado conjugal, religião, ocupação, escolaridade) e econômicos (renda mensal familiar em salários mínimos - SM). Também foi utilizado um questionário estruturado contendo questões referentes a autopercepção do estado geral de saúde do paciente, condições gerais de saúde, comorbidades (hipertensão arterial sistêmica - HAS, diabetes mellitus DM, déficit visual, doença osteoarticular e outras doenças crônicas) e uso regular de medicamentos.

O estado cognitivo foi avaliado por meio do MEEM. Considerando as influências do grau de escolaridade no escore final do MEEM, foram adotados os seguintes pontos de corte: 20 pontos para analfabetos; 25 para pessoas com um a quatro anos de escolaridade; 26,5 para aquelas com cinco a oito anos de escolaridade; 28 para indivíduos que estudaram de nove a 11 anos e 29 para aqueles com escolaridade superior a 11 anos (Brucki et al., 2003).

Os dados referentes a queda e ao medo de cair foram coletados por meio de um questionário estruturado, utilizando as seguintes perguntas: "Nos últimos 12 meses, você sofreu quantas quedas?"; "Você deixou de realizar alguma atividade (física ou de lazer) por medo de cair?”. Para a questão "Nos últimos 12 meses, você sofreu quantas quedas?”, se o paciente respondeu uma ou mais quedas foi considerado "sim” para ocorrência de quedas. Para as demais questões as respostas foram categóricas, "sim" ou "não".

O risco de queda foi avaliado por meio do teste Timed Up and Go (TUG) que mede em segundos, o ato de um 
indivíduo levantar de uma cadeira sem braços (altura de aproximadamente 46 centímetros), caminhar uma distância de 3 metros, virar, caminhar de volta para a cadeira e sentar-se novamente (Podsiadlo et al., 1991). A realização do teste em até 10 segundos é considerada normal para adultos saudáveis, independentes e sem risco de quedas; valores entre 11 e 20 segundos é o esperado para idosos com deficiência ou frágeis, com independência parcial e com baixo risco de quedas; acima de 20 segundos sugere que o idoso apresenta importante déficit da mobilidade física e risco de quedas, sendo esperado que idosos comunitários tenham um desempenho de até 12 segundos na realização do teste (Bischoff et al., 2003). Nesse estudo foi considerado o tempo de até 10 segundos para indivíduos sem risco de queda, 11 a 20 segundos para risco de queda baixo/moderado e valores acima de 20 segundos como risco importante de quedas.

Quanto aos procedimentos de divulgação, recrutamento dos participantes e coleta de dados, inicialmente ocorreu a divulgação da pesquisa na UBS, na qual o estudo foi conduzido, em centros de convivência de idosos no município de realização da pesquisa e por meio de mídias eletrônicas. Foi realizada a entrega da carta explicativa e do TCLE para os idosos que se interessaram em participar do estudo. Em seguida, foi agendada a data para a avaliação. No dia da coleta de dados os voluntários passaram por entrevista, sendo preenchidos os questionários referentes às características socioeconômicas, ao estado geral de saúde, o MEEM e o questionário sobre o histórico de quedas e medo de cair. Todos os questionários foram aplicados por meio de entrevista conduzida por pesquisadora previamente treinada, em ambiente reservado. Na sequência foi realizado o teste TUG, sendo executada uma tentativa inicial para familiarização, sem cronometrar o tempo, e posteriormente a realização do teste com o registro do tempo de execução.

No final da coleta foi conduzida a avaliação antropométrica, com aferição da massa corporal total e da estatura, utilizando uma balança digital portátil HBF-214 (OMRON Healthcare, Inc., Lake Forest, IL, Estados Unidos da América) com precisão de 0,1 kg e capacidade máxima de $150 \mathrm{~kg}$ e estadiômetro portátil (Sanny, São Bernardo do Campo, SP, Brasil) com precisão de $0,1 \mathrm{~cm}$ e extensão máxima de 2,20 metros. O índice de massa corporal (IMC) foi calculado por meio da fórmula: massa corporal total $(\mathrm{kg}) / \mathrm{estatura}(\mathrm{m})^{2}$.

Os dados foram analisados com auxílio do Statistical Package for Social Sciences (SPSS Inc., Chicago, IL, EUA, Release 16.0.2, 2008), após inserção por meio de dupla digitação. Inicialmente, foi verificada a normalidade dos dados contínuos pelo teste de Shapiro-Wilk. Foi realizada análise descritiva para as variáveis categóricas e distribuição das frequências absolutas e relativas e para as variáveis numéricas calculados a mediana, valor mínino (Valor ${ }_{\text {min }}$ ) e valor máximo (Valor ${ }_{\text {máx }}$. $\mathrm{Na}$ análise inferencial foram calculadas as associações entre a variável dependente (ocorrência de quedas) e cada variável independente (idade, sexo, comorbidades, risco de queda, e medo de cair), por meio dos testes qui-quadrado $\left(X^{2}\right)$ ou exato de Fisher. Os intervalos de confiança de $95 \%$ ( IC $_{95 \%}$ ) também foram calculados. Em todas as análises foi adotado um nível de significância bilateral de 5\%.

\section{Resultados}

Foram avaliados 40 idosos, dois foram excluídos pois não aguardaram a conclusão do preenchimento dos questionários, totalizando ao final 38 indivíduos analisados. A distribuição das características sociodemográficas e econômicas dos idosos avaliados estão apresentadas na Tabela 1. 
Tabela 1. Características sociodemográficas e econômicas autorrelatadas por idosos adscritos a uma Unidade Básica de Saúde da zona urbana do município de Petrolina, PE, Brasil $(\mathrm{n}=38)$

\begin{tabular}{|c|c|c|c|}
\hline Variáveis & $\mathbf{n}$ & $\%$ & $\mathrm{IC}_{95 \%}$ \\
\hline \multicolumn{4}{|l|}{$\overline{\text { Sexo }}$} \\
\hline Feminino & 28 & 73,7 & $57,99-85,03$ \\
\hline Masculino & 10 & 26,3 & $14,97-42,01$ \\
\hline \multicolumn{4}{|l|}{ Ocupação } \\
\hline Aposentado & 30 & 78,9 & $63,65-88,93$ \\
\hline Trabalha & 3 & 7,9 & $2,72-20,80$ \\
\hline Outra & 5 & 13,2 & $5,75-27,33$ \\
\hline \multicolumn{4}{|l|}{ Religião } \\
\hline Católica & 34 & 89,5 & $75,87-95,83$ \\
\hline Evangélica & 4 & 10,5 & $4,17-24,13$ \\
\hline \multicolumn{4}{|l|}{ Estado conjugal } \\
\hline Solteiro & 6 & 15,8 & $7,44-30,42$ \\
\hline Casado ou união estável & 17 & 44,7 & $30,15-60,29$ \\
\hline Viúvo & 9 & 23,7 & $12,99-39,21$ \\
\hline Outro & 6 & 15,8 & $7,44-43,71$ \\
\hline \multicolumn{4}{|l|}{ Escolaridade } \\
\hline Analfabeto ou fundamental incompleto & 16 & 42,1 & $27-85-57,81$ \\
\hline Fundamental completo ou médio incompleto & 8 & 21,0 & $11,07-36,35$ \\
\hline Médio completo ou superior incompleto & 8 & 21,0 & $11,07-36,35$ \\
\hline Superior completo & 6 & 15,8 & $7,44-43,71$ \\
\hline \multicolumn{4}{|l|}{ Renda mensal em salários mínimos (SM)* } \\
\hline Menos de $1 \mathrm{SM}$ & 5 & 13,2 & $5,75-27,33$ \\
\hline 1 a $2 \mathrm{SM}$ & 27 & 71,0 & $55,24-83,00$ \\
\hline Maior que $2 \mathrm{SM}$ & 6 & 15,8 & $7,44-43,71$ \\
\hline
\end{tabular}

*Salário mínimo baseado no valor de $\mathrm{R} \$ 954,00$ (coleta de dados realizada no ano de 2018).

Fonte: Autores.

A mediana (Valor ${ }_{\text {min }}$ - Valor ${ }_{\text {máx }}$ ) da idade foi de 68,5 (60,0 - 92,0) anos. Em relação às variáveis antropométricas a mediana da massa corporal foi de 68,2 $\mathrm{kg}(40,8$ - 94,0), a de estatura 1,55 m (1,42 - 1,85) e o IMC apresentou mediana de 68,5 $\mathrm{kg} / \mathrm{m}^{2}(60,0-92,0)$.

Dos avaliados, 78,9\% $(\mathrm{n}=30)\left(\mathrm{IC}_{95 \%} 63,65\right.$ - 88,93) responderam que apresentavam alguma doença crônica. Em relação ao diagnóstico de doença osteoarticular, 47,4\% $(\mathrm{n}=18)\left(\mathrm{IC}_{95 \%}\right.$ 32,48 - 62,74) referiram que tinham algum problema relacionado e 68,4 \% $(\mathrm{n}=26)\left(\mathrm{IC}_{95 \%} 52,54\right.$ - 80,92) relataram presença de déficit visual. Na Tabela 2 estão apresentadas a distribuição das frequências relacionadas a autopercepção do estado geral de saúde do paciente, comorbidades e uso de medicamentos dos idosos avaliados. 
Tabela 2. Características relacionadas a autopercepção do estado geral de saúde, comorbidades e uso de medicamentos em idosos adscritos a uma Unidade Básica de Saúde da zona urbana do município de Petrolina, PE, Brasil $(\mathrm{n}=38)$

\begin{tabular}{lccc}
\hline Variáveis & $\mathbf{n}$ & $\boldsymbol{\%}$ & IC95\% \\
\hline Como considera a sua saúde & 12 & 31,6 & $19,08-47,46$ \\
Muito boa ou boa & 22 & 57,9 & $42,19-72,15$ \\
Regular & 4 & 10,5 & $4,17-24,13$ \\
Ruim ou muito ruim & & & $44,72-74,40$ \\
Comorbidades & 23 & 60,5 & $23,38-52,72$ \\
Hipertensão Arterial Sistêmica & 14 & 36,8 & $69,58-92,56$ \\
Diabetes Mellitus & & & $7,44-30,42$ \\
Uso de medicamentos & 32 & 84,2 & 15,8 \\
Sim & 6 & & \\
Não & & & \\
\hline
\end{tabular}

Fonte: Autores.

Em relação ao escore geral do MEEM, nenhum dos avaliados apresentou pontuação menor que os pontos de corte por escolaridade e a mediana (Valor ${ }_{\text {min }}$ - Valor máx $_{\text {) foi }} 24(15,0$ - 30,0). Quanto ao teste TUG, a mediana de tempo para realização do testes foi 11,0 segundos $(7,0$ - 20,0). Com base na classificação do risco de queda pelo TUG, nenhum idoso avaliado apresentou risco elevado de queda. Quanto à ocorrência de quedas nos últimos 12 meses, dos avaliados 42,1\% (n = 16) (IC $95 \%$ $27,85$ - 57,81) sofreram uma queda ou mais e 21,1\% ( $\mathrm{n}=8)\left(\mathrm{IC}_{95 \%} 11,07\right.$ - 36,35) deixaram de realizar alguma atividade por medo de cair.

A associação entre a ocorrência de queda e as variáveis categóricas idade, sexo, doença crônica, risco de queda e medo de cair dos idosos é demonstrada na Tabela 3. 
Tabela 3. Associação entre ocorrência de queda e variáveis idade, sexo, doença crônica, risco de queda e medo de cair em idosos adscritos a uma Unidade Básica de Saúde da zona urbana de Petrolina, PE, Brasil $(\mathrm{n}=38)$

\begin{tabular}{|c|c|c|c|}
\hline \multirow[b]{2}{*}{ Variáveis } & \multicolumn{2}{|c|}{ Ocorrência de queda no último ano } & \multirow[b]{2}{*}{$\mathbf{P}$} \\
\hline & $\begin{array}{c}\text { Nenhuma queda } \\
\text { n }(\%)\end{array}$ & $\begin{array}{c}\text { Uma ou mais quedas } \\
\text { n(\%) }\end{array}$ & \\
\hline \multicolumn{4}{|l|}{ Idade } \\
\hline $60-65$ & $8(72,7)$ & $3(27,3)$ & \\
\hline $66-75$ & $12(54,5)$ & $10(45,5)$ & $0,193^{*}$ \\
\hline$\geq 76$ & $2(40,0)$ & $3(60,0)$ & \\
\hline \multicolumn{4}{|l|}{ Sexo } \\
\hline Feminino & $16(57,1)$ & $12(42,9)$ & \\
\hline Masculino & $6(60,0)$ & $4(40,0)$ & $1,00^{*}$ \\
\hline \multicolumn{4}{|l|}{ Doença crônica ${ }^{\dagger}$} \\
\hline Sim & $13(48,1)$ & $14(51,9)$ & \\
\hline Não & $9(81,8)$ & $2(18,2)$ & $0,078^{*}$ \\
\hline \multicolumn{4}{|l|}{ Risco de queda (TUG) $)^{\dagger \dagger}$} \\
\hline Sem risco & $10(62,5)$ & $6(37,5)$ & \\
\hline Risco baixo a moderado & $12(57,1)$ & $9(42,9)$ & $1,00 * *$ \\
\hline \multicolumn{4}{|l|}{ Medo de cair } \\
\hline Sim & $3(37,5)$ & $5(62,5)$ & \\
\hline Não & $19(63,3)$ & $11(36,7)$ & $0,243 *$ \\
\hline \multicolumn{4}{|l|}{ Déficit visual } \\
\hline Sim & $13(50,0)$ & $13(50,0)$ & \\
\hline Não & $9(75,0)$ & $3(25,0)$ & $0,178^{*}$ \\
\hline \multicolumn{4}{|l|}{ Doença osteoarticular } \\
\hline Sim & $8(44,4)$ & $10(55,6)$ & \\
\hline Não & $13(76,5)$ & $4(23,5)$ & $0,086^{*}$ \\
\hline
\end{tabular}

TUG: Timed Up and Go; †Hipertensão arterial sistêmica e/ou diabetes mellitus; ${ }^{\dagger}$ Dado ausente de um voluntário; *Teste exato de Fisher; **Teste qui-quadrado de Pearson

Fonte: Autores

\section{Discussão}

O presente estudo identificou uma frequência elevada de ocorrência de quedas entre os idosos avaliados $(42,1 \%)$, no entanto os resultados do teste TUG mostraram que a amostra avaliada não apresentou alto risco de queda. Em adição, não foi observada associação significativa entre a ocorrência de queda e as variáveis analisadas: idade, sexo, doença crônica (HAS e/ou DM), risco de queda, medo de cair, déficit visual e doença osteoarticular.

Quanto às características da amostra foi observada maior prevalência de mulheres entre os avaliados. Fato comum em estudos realizados em serviços de saúde pois esses espaços são culturalmente vistos como locais de mulheres. Um estudo nacional demonstrou que as mulheres buscam por serviços de saúde aproximadamente duas vezes mais que os homens (Levorato et al., 2014).

Em relação à renda e à escolaridade dos participantes, $84,2 \%$ tinham renda mensal de até dois salários mínimos e 
42,1\% eram analfabetos ou não tinham concluído o ensino fundamental. Dados de outras pesquisas evidenciaram que mais de $50 \%$ dos idosos brasileiros possuem baixa renda e pouca escolaridade (Borges et al., 2014). Fatores que combinados podem contribuir para maior ocorrência de quedas devido aspectos ambientais e questões relacionados ao cuidado com a saúde de forma geral (Abreu et al., 2016).

Outra variável analisada neste estudo foi a autopercepção de saúde, que é entendida como indicador geral de saúde e preditor relevante de incapacidade funcional em idosos (Antunes et al., 2018; Borges et al., 2014). Na amostra avaliada foi verificado que 31,6\% consideraram seu estado de saúde muito bom, 57,9\% consideravam como regular e 10,5\% como ruim ou muito ruim. Apesar disso, 78,9\% dos idosos relataram ter o diagnóstico de pelo menos uma doença crônica e a maioria relatou fazer uso regular de medicamentos. Essa disparidade pode ser justificada devido a percepção de saúde estar mais relacionada às incapacidades do que à presença de doenças crônicas (Borges et al., 2014).

Em relação à ocorrência de quedas, foi observado no presente estudo que $42,1 \%$ dos idosos entrevistados relataram episódios de uma ou mais quedas nos últimos 12 meses. Resultados semelhantes a outros estudos realizados com idosos brasileiros (Motta et al., 2010; Nascimento \& Tavares, 2016; Santos et al., 2015; Vieira et al., 2018). Por outro lado, no estudo de Prato et al. (2017) foi observada uma frequência menor de quedas $(24,3 \%)$, o que pode estar relacionado ao fato de tal estudo ter avaliado indivíduos a partir dos 55 anos de idade.

Cabe ressaltar outra variável importante na atenção à saúde da pessoa idosa, o medo de cair, uma vez que pode influenciar nas atividades de vida diária (AVD), além de prejudicar o bem-estar físico e psicossocial dos indivíduos (Rivasi et al., 2020). Dentre os avaliados, a frequência de indivíduos que deixaram de realizar alguma atividade por medo de cair foi baixa, quando comparada aos dados expressos na literatura, de que até $85 \%$ dos idosos podem apresentar medo de cair (Scheffer et al., 2008).

De acordo com a análise do resultado do teste TUG observou-se que a população estudada não apresentou alto risco de queda. O tempo normal de realização do teste para idosos que vivem na comunidade é de até 12 segundos (Bischoff et al., 2003), uma boa mobilidade funcional indicada pelo teste TUG pode significar melhor desempenho musculoesquelético e capacidade funcional (Wamser et al., 2015). Apesar disso, cabe ressaltar que a prevalência de quedas no último ano foi elevada apesar de nenhum avaliado da amostra apresentar alto risco de queda, sendo importante que medidas preventivas de quedas sejam implementadas para todos os idosos.

A queda é considerada um evento multifatorial, contudo, no presente estudo não foi observada associação significativa com as variáveis sexo, idade, comorbidades, risco de queda, medo de cair, déficit visual e doença osteoarticular. Na literatura as mulheres apresentam uma maior ocorrência de quedas (Nascimento \& Tavares, 2016; Rodrigues et al., 2014), e é demonstrado que as mesmas têm maiores chances de apresentar lesões devido as quedas do que os homens (Boyd et al., 2009). Essa relação pode ser parcialmente explicada pelas mudanças hormonais, relacionadas à menopausa e as diferenças estruturais como a proporção de massa isenta de gordura (Bekibele et al., 2010; Smith et al., 2017). O que pode justificar a prevalência elevada de quedas na presente pesquisa, uma vez que a amostra foi predominantemente feminina.

Contrariamente aos resultados identificados, um estudo populacional com idosos brasileiros evidenciou associação entre doenças osteoarticulares e histórico de quedas (Rodrigues et al., 2014). Além disso, observa-se que a presença de morbidades e o uso de muitos medicamentos também apresentaram associação com a ocorrência de quedas (Nascimento \& Tavares, 2016).

Santos et al. (2015) de forma semelhante ao observado neste estudo não encontraram associação entre presença de déficit visual e ocorrência de quedas em idosos. Por outro lado, o déficit visual é uma variável que normalmente possui relação com incidência de quedas, pois pode interferir na execução das atividades diárias. Szabo et al. (2008) relatam que dois terços dos pacientes com problemas relacionados a degeneração da idade macular apresentaram déficits visuomotores e de equilíbrio, 
resultando em falta de mobilidade e um risco aumentado de quedas. Nesse mesmo estudo, foi relatado que a perda do campo visual aumenta o risco de quedas.

Apesar da maioria dos avaliados apresentar HAS, DM, doenças osteoarticulares e déficit visual, no presente estudo não foi verificada associação significativa entre essas condições de saúde e ocorrência de quedas no último ano. No entanto, tais resultados podem ser decorrentes das limitações apresentadas nesta pesquisa, tais como o tamanho e características da amostra, bem como o viés de memória, pois os questionários utilizados para avaliação baseiam-se em questões autorreferidas e as pessoas idosas podem apresentar dificuldade de lembrar alguns eventos após um período de tempo de sua ocorrência.

Portanto, sugere-se a realização de outros estudos que avaliem uma amostra populacional de idosos da zona urbana e da zona rural que representem grupos distintos em relação à capacidade funcional, e que contemplem fatores de riscos ambientais e comportamentais. Uma vez que a prevenção de quedas entre os idosos é uma importante questão de saúde pública (Park, 2018), os resultados de estudos acerca dessa temática são de fundamental importância pois podem subsidiar a elaboração e desenvolvimento de medidas de promoção e de prevenção à saúde da população idosa.

\section{Conclusão}

A frequência de quedas foi elevada entre os idosos adscritos a uma Unidade Básica de Saúde do município de Petrolina, sertão do estado de Pernambuco, Brasil. No entanto, não foi observada associação estatística entre ocorrência de queda e os fatores comumente preditivos: idade, sexo, doença crônica (HAS e/ou DM), risco de queda, medo de cair, déficit visual e doença osteoarticular.

\section{Agradecimentos}

Agradecemos aos idosos que participaram deste estudo. O presente trabalho foi realizado com apoio da Coordenação de Aperfeiçoamento de Pessoal de Nível Superior - Brasil (CAPES) - Código de Financiamento 001. E financiamento da Fundação de Amparo à Ciência e Tecnologia do Estado de Pernambuco - FACEPE, por meio de concessão de bolsa para o Programa de Pós-Graduação.

\section{Referências}

Abreu, D. R. de O. M., Azevedo, R. C. de S., da Silva, A. M. C., Reiners, A. A. O., \& Abreu, H. C. A. (2016). Factors associated with recurrent falls in a cohort of older adults. Ciência \& Saúde Coletiva, 21(11), 3439-3446. https://doi.org/10.1590/1413-812320152111.21512015

Almeida-Santos, M. A., Barreto-Filho, J. A., Oliveira, J. L. M., Reis, F. P., da Cunha Oliveira, C. C., \& Sousa, A. C. S. (2016). Aging, heart rate variability and patterns of autonomic regulation of the heart. Archives of Gerontology and Geriatrics, 63, 1-8. https://doi.org/10.1016/j.archger.2015.11.011

Antunes, J. L. F., Chiavegatto Filho, A. D. P., Duarte, Y. A. O., \& Lebrão, M. L. (2018). Desigualdades sociais na autoavaliação de saúde dos idosos da cidade de São Paulo. Revista Brasileira de Epidemiologia, 21(suppl 2), 1-14. https://doi.org/10.1590/1980-549720180010.supl.2

Bekibele, C. O., \& Gureje, O. (2010). Fall Incidence in a Population of Elderly Persons in Nigeria. Gerontology, 56(3), 278-283. https://doi.org/10.1159/000236327

Bischoff, H. A., Stähelin, H. B., Monsch, A. U., Iversen, M. D., Weyh, A., von Dechend, M., Akos, R., Conzelmann, M., Dick, W., \& Theiler, R. (2003). Identifying a cut-off point for normal mobility: A comparison of the timed "up and go" test in community-dwelling and institutionalised elderly women. Age and Ageing, 32(3), 315-320. https://doi.org/10.1093/ageing/32.3.315

Borges, A. M., Santos, G., Kummer, J. A., Fior, L., Molin, V. D., \& Wibelinger, L. M. (2014). Autopercepção de saúde em idosos residentes em um município do interior do Rio Grande do Sul. Revista Brasileira de Geriatria e Gerontologia, 17(1), 79-86. https://doi.org/10.1590/S1809-98232014000100009

Boyd, R., \& Stevens, J. A. (2009). Falls and fear of falling: burden, beliefs and behaviours. Age and Ageing, 38(4), 423-428. https://doi.org/10.1093/ageing/afp053

Brucki, S. M. D., Nitrin, R., Caramelli, P., Bertolucci, P. H. F., \& Okamoto, I. H. (2003). Sugestões para o uso do mini-exame do estado mental no Brasil. Arquivos de Neuro-Psiquiatria, 61(3 B), 777-781. https://doi.org/10.1590/S0004-282X2003000500014 
Gamage, N., Rathnayake, N., \& Alwis, G. (2019). Prevalence and associated risk factors of falls among rural community-dwelling older people: a crosssectional study from southern Sri Lanka. Current Gerontology and Geriatrics Research, 2019, 2370796. https://doi.org/10.1155/2019/2370796

Levorato, C. D., Mello, L. M. de, Silva, A. S. da, \& Nunes, A. A. (2014). Fatores associados à procura por serviços de saúde numa perspectiva relacional de gênero. Ciência \& Saúde Coletiva, 19(4), 1263-1274. https://doi.org/10.1590/1413-81232014194.01242013

Miranda, G. M. D., Mendes, A. da C. G., \& Silva, A. L. A. da. (2016). Population aging in Brazil: current and future social challenges and consequences. Revista Brasileira de Geriatria e Gerontologia, 19(3), 507-519. https://doi.org/10.1590/1809-98232016019.150140

Motta, L. B. da, Aguiar, A. C. de, Coutinho, E. S. F., \& Huf, G. (2010). Prevalência e fatores associados a quedas em idosos em um município do Rio de Janeiro. Revista Brasileira de Geriatria e Gerontologia, 13(1), 83-91. https://doi.org/10.1590/S1809-98232010000100009

Nascimento, J. S., \& Tavares, D. M. dos S. (2016). Prevalência e fatores associados a quedas em idosos. Texto \& Contexto - Enfermagem, $25(2)$, e0360015. https://doi.org/10.1590/0104-07072016000360015

Park, S. H. (2018). Tools for assessing fall risk in the elderly: a systematic review and meta-analysis. Aging Clinical and Experimental Research, 30(1), 1-16. https://doi.org/10.1007/s40520-017-0749-0

Pereira A. S.; Shitsuka D. M.; Parreira F. J., \& Shitsuka R. (2018). Metodologia da pesquisa científica. UAB/NTE/UFSM.

Podsiadlo, D., \& Richardson, S. (1991). The timed "Up \& Go": a test of basic functional mobility for frail elderly persons. Journal of the American Geriatrics Society, 39(2), 142-148. https://doi.org/10.1111/j.1532-5415.1991.tb01616.x

Prato, S. C. F., Andrade, S. M. de, Cabrera, M. A. S., Dip, R. M., Santos, H. G. dos, Dellaroza, M. S. G., \& Mesas, A. E. (2017). Frequency and factors associated with falls in adults aged 55 years or more. Revista de Saúde Pública, 51, 37. https://doi.org/10.1590/s1518-8787.2017051005409

Rivasi, G., Kenny, R. A., Ungar, A., \& Romero-Ortuno, R. (2020). Predictors of incident fear of falling in community-dwelling older adults. Journal of the American Medical Directors Association, 21(5), 615-620. https://doi.org/10.1016/j.jamda.2019.08.020

Rodrigues, I. G., Fraga, G. P., \& Barros, M. B. de A. (2014). Falls among the elderly: risk factors in a population-based study. Revista Brasileira de Epidemiologia, 17(3), 705-718. https://doi.org/10.1590/1809-4503201400030011

Santos, R. K. M. dos, Maciel, Á. C. C., Britto, H. M. J. de S., Lima, J. C. C., \& Souza, T. O. de. (2015). Prevalência e fatores associados ao risco de quedas em idosos adscritos a uma Unidade Básica de Saúde do município de Natal, RN, Brasil. Ciência \& Saúde Coletiva, 20 (12), 3753-3762. https://doi.org/10.1590/1413-812320152012.00662015

Santos, M. A. A., Sousa, A. C. S., Reis, F. P., Santos, T. R., Lima, S. O., \& Barreto-Filho, J. A. (2013). Envelhecer altera relevantemente a frequência cardíaca média? Arquivos Brasileiros de Cardiologia, 101(5), 388-398. https://doi.org/10.5935/abc.20130188

Scheffer, A. C., Schuurmans, M. J., van Dijk, N., van der Hooft, T., \& de Rooij, S. E. (2008). Fear of falling: measurement strategy, prevalence, risk factors and consequences among older persons. Age and Ageing, 37(1), 19-24. https://doi.org/10.1093/ageing/afm169

Siqueira, F. V., Facchini, L. A., Silveira, D. S. da, Piccini, R. X., Tomasi, E., Thumé, E., Silva, S. M., \& Dilélio, A. (2011). Prevalence of falls in elderly in Brazil: a countrywide analysis. Cadernos de Saúde Pública, 27(9), 1819-1826. https://doi.org/10.1590/S0102-311X2011000900015

Smith, A. de A., Silva, A. O., Rodrigues, R. A. P., Moreira, M. A. S. P., Nogueira, J. de A., \& Tura, L. F. R. (2017). Assessment of risk of falls in elderly living at home. Revista Latino-Americana de Enfermagem, 25. https://doi.org/10.1590/1518-8345.0671.2754

Szabo, S. M., Janssen, P. A., Khan, K., Potter, M. J., \&Lord, S. R. (2008). Older women with age-related macular degeneration have a greater risk of falls: a physiological profile assessment study. Journal of the American Geriatrics Society, 56(5), 800-807. https://doi.org/10.1111/j.1532-5415.2008.01666.x

Teixeira, D. K. da S., Andrade, L. M., Santos, J. L. P., \& Caires, E. S. (2019). Falls among the elderly: environmental limitations and functional losses. Revista Brasileira de Geriatria e Gerontologia, 22(3), 1-10. https://doi.org/10.1590/1981-22562019022.180229

Vieira, L. S., Gomes, A. P., Bierhals, I. O., Farías-Antúnez, S., Ribeiro, C. G., Miranda, V. I. A., Lutz, B. H., Barbosa-Silva, T. G., Lima, N. P., Bertoldi, A. D., \& Tomasi, E. (2018). Falls among older adults in the South of Brazil: prevalence and determinants. Revista de Saúde Pública, 52, 22. https://doi.org/10.11606/S1518-8787.2018052000103

Wamser, E. L., Valderramas, S. R., Paula, J. A. de, Schieferdecker, M. E. M., Amarante, T. P., Pinotti, F., Coelho, R. A., Stanczk, L., Guimarães, A. T. B., \& Gomes, A. R. S. (2015). Melhor desempenho no teste timed up and go está associado a melhor desempenho funcional em idosas da comunidade. Geriatrics Gerontology Aging, 9(2), 138-143. https://doi.org/10.5327/Z2447-2115201500040003 